tion which suggest that the author has not quite achieved a thorough understanding of the subject. Thus, in respect of Mendel's peas, we are told that it takes at least two generations of self-fertilisation in order to establish whether or not a tall individual is true-breeding for this character (frame 51 ). Again, in frame 146 and thereafter, the association of two chromatids at first meiotic anophase are referred to as bivalents, a term usually restricted to the association of homologous chromosomes at diplotene, diakinesis and metaphase. Such errors could have been removed from the text had a competent geneticist been asked to read it before publication.

It is less easy to understand, however, how infuriating errors of other types have remained undetected. Thus frame 43 shows a diagram which indicates that all tall $F_{2}$ pea plants in Mendel's experiment segregate in $F_{3}$, which is in flat contradiction to the written evidence of the previous frame. There are also a number of "slips" and other typographical errors. Yet this text, as is common practice, was subjected to a validation test before publication, though we are provided with insufficient details of this test in respect to numbers of students and their educational attainment. It is, therefore, difficult to take such a validation certificate seriously.

Despite these criticisms this book, when it is revised, will be a very welcome addition to the ranks of inexpensive introductory texts on genetics and I am disappointed that it has not been more carefully produced.

M. J. Lawrence.

\title{
COMPLEMENTATION: FACTS, THEORIES AND SPECULATIONS
}

GENETIC COMPLEMENTATION. J. R. S. Fincham. W. A. Benjamin Inc., New York, 1966. Pp. xiit143. $\$ 9.65$.

This book is a concise summary of the extensive literature on genetic complementation written for advanced students, research workers and those who teach biochemical genetics. It opens with a comparative account of the genetic systems which have been used for complementation studies in higher organisms, fungi, bacteria and bacteriophages. In spite of the great diversity of systems used in these studies the author rather surprisingly concludes that they all give equivalent information, at least at the qualitative level.

In the next two chapters the approach is basically historical. We are taken from the earliest definition of a gene to the one cistron-one polypeptide chain hypothesis in chapter 2, and from the breakdown of the original cistron concept to the emergence of the operon hypothesis in chapter 3 . In chapter 2 and for the remainder of the book the term gene is used as synonymous with cistron.

The two most favoured of the possible mechanisms of interallelic complementation, namely, reassortment of gene products and hybrid protein production, are considered in chapter 4 . Both the evidence presented, and the author, clearly favour the latter interpretation, the superiority of the hybrid protein being explained by the correction of the conformation of the protein molecule.

Chapter 5 is concerned with complementation maps and their interpretation. Following a survey of the incidence of linear, circular and more complex maps and their structural meaning, the author takes a firm line about the value of the effort that is put into the construction of elaborate comple- 
mentation maps. While admitting their usefulness as a means of condensing many data into a compact diagram he points out their limited value unless there is correspondingly detailed information about the three-dimensional structure of the protein product.

The final chapter is devoted to speculations about the possible evolutionary significance of allelic complementation. Significance is sought in the overdominance theory of heterosis. In the thirty years since this theory first began to receive serious consideration there has been a singular lack of unambiguous evidence of its validity although there have been a number of demonstrations of its inapplicability to specific cases of heterosis. In support of the theory the author repeats conclusions current in the late forties (the most recent reference quoted being 1952) that are no longer valid. But leaving this aside the speculations founder on the complete lack of evidence that hybrid proteins have any functional superiority.

A problem that the author could have profitably considered in this chapter is why, if allelic complementation has evolutionary significance rather than being an "instructive artifact", it has become so highly developed in lower organisms whose whole vegetative existence usually takes place in the haploid state.

In a book so full of ideas the reader is bound to find himself in disagreement with the author over some of the more speculative content but this in no way detracts from the fact that "Genetic Complementation" is a critical, lucid and comprehensive review of a subject that occupies a central position in present-day genetics. It is well worth anyone's time and money.

$$
\text { J. L. Jinks. }
$$

\section{MATRICES WITHOUT TEARS}

MATRIX ALGEBRA FOR THE BIOLOGICAL SCIENCES (Including Applications in Statistics). S. R. Searle. John Wiley \& Sons, New York, 1966. Pp. xii +296. 75s.

To biologists, the need for a comprehensive text on matrix algebra has been apparent for some time. Matrices are increasingly used in the quantitative branches of biology, notably in population genetics and biometrical genetics, and are an essential part of any advanced course in these subjects. Dr. Searle's book is therefore welcome, and deserves particular attention.

The book opens with a description of matrices and the notation involved, elementary matrix operations, determinants and expansions. It then proceeds to discuss inversion of matrices and some aspects of the matrix theory such as rank and linear independence. These are followed by accounts of generalized inversed matrices and latent roots and vectors. A chapter is devoted to " miscellanea ", including such topics as inversion by partitioning, differential operators and direct sums and products of matrices. The two last chapters are devoted to statistical techniques which are introduced via the matrix terminology: least square estimation, linear and multilinear regression, analysis of variance, linear models and normal equations.

The text is lucidly written, the notations usually clear, and relatively little previous knowledge of algebra beyond school level is required. This last characteristic will probably add to the book's appeal to biologists. Many numerical examples are given as well as illustrations from statistics and biology. The majority of the latter are genetical problems; for instance, generation matrices under different systems of matings and estimation of 